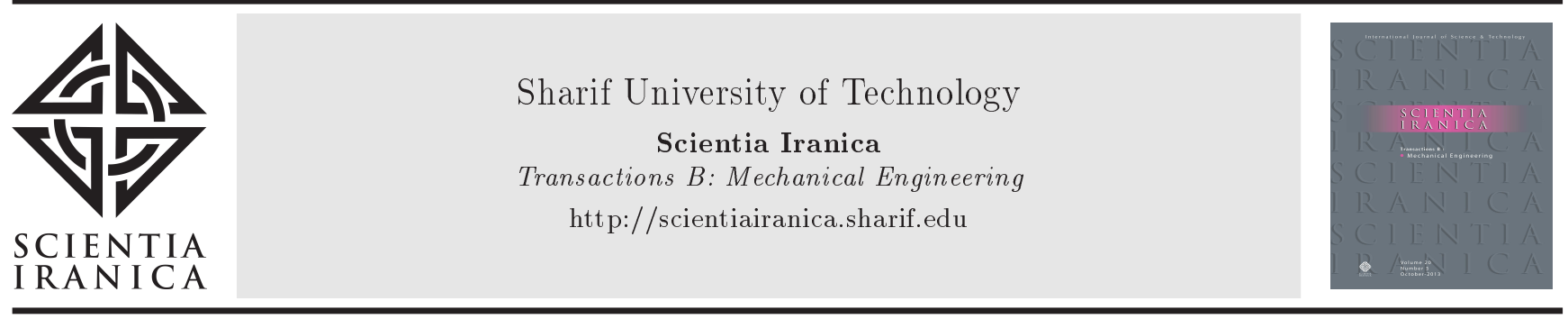

\title{
Parametric resonance domain of a parametric excited screen machine
}

\author{
M. Zahedi ${ }^{\mathrm{a}}$, I. Khatami ${ }^{\mathrm{b}}$, and S.A. Zahedic,* \\ a. Department of Computer Engineering, University of Isfahan, Isfahan, Iran. \\ b. Department of Mechanical Engineering, Chabahar Maritime University, Chabahar, Iran. \\ c. School of Engineering and Sustainable Development, De Montfort University, Leicester, UK.
}

Received 14 February 2019; received in revised form 2 October 2019; accepted 29 June 2020

\section{KEYWORDS}

Parametric Resonance

(PR);

Homotopy

Perturbation Method

(HPM);

Screen machine;

Impact analysis;

Mineral science.

\begin{abstract}
In this paper, the stable operation domain of a Parametric Resonance (PR) based screen machine is thoroughly investigated. The dynamic model of a screen with two differential equations coupled by a geometric nonlinearity is applied to study the structural motion. In order to address the strong nonlinearities in the equations of motion, the Homotopy Perturbation Method (HPM) is used to compute the longitudinal and transverse oscillations. Since, under excessive excitation, the contact of the vibration screen and loaded materials displays an undesirable vibro-impact response, the HPM is applied under impact and non-impact operation conditions. By considering appropriate technological parameters, including the maximum amplitude desired, spring stiffness, screen mass, initial velocity and acceleration, the best domain of excitation is calculated. The results provide the optimal parameter domain for the new design of a vibrating screen, where the parametric oscillations are excited and the analytically obtained steady oscillation regime is stable. The outcomes of this research respond to changes in the production quality and quantity that are required by customers, and deliver a design guideline for engineers.
\end{abstract}

(C) 2021 Sharif University of Technology. All rights reserved.

\section{Introduction}

A vibrating screen is a type of equipment used for the large-scale separation and classification of solid particles by size. It is widely used in many engineering industries such as metallurgy, food, medicine, mining, artificial sand production, and agriculture $[1,2]$. The components of screen machines in principle include a fixed base, a perforated screen mounted for movement relative to the base during operation, vibrating motors and springs [3]. Designing screen-working parameters

*. Corresponding author. Tel.: +44 (0)116-201-3971 E-mail address: Abolfazl.Zahedi@dmu.ac.uk (S.A. Zahedi)

doi: $10.24200 /$ sci. 2020.52943 .2959 is a great challenge, since the vibration should produce an exciting force that crosses the mass centre to avoid material blockage [4].

In a commercial screen machine, sieve efficiency is an essential evaluation indicator of performance and, therefore, many researchers try to improve it [57]. While Parametric Resonance (PR) is mainly cited as an undesirable phenomenon in shell structures [8], MEMS/NEMS devices [9], and nanowire systems [10], etc., L. Slepyan and V. Slepyan [11] invented a new type of vibrating screen that is parametrically excited. This design eliminates many of the drawbacks of existing screens used for naturally wet mineral materials, such as aggregates, in production, and provides the quantity demanded by the customers. A PRbased screen machine generates high impact energy 

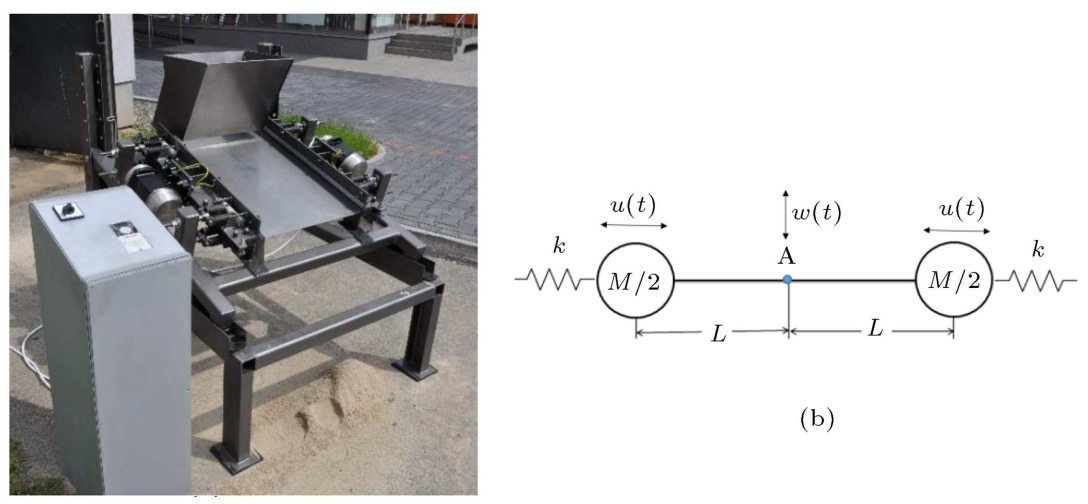

(b)

(a)

Figure 1. PR-based vibration screen (a) and its dynamic model (b) after model of L. Slepyan and V. Slepyan [11].

by increasing the vibration amplitude significantly and compares favourably with conventional size-sorting machines in which transverse oscillations are excited directly $[12,13]$. Exciting the sieve by parametric vibration results in the crushing of glutted grains of material and the degreasing of the adhesion force between the material and the sieve. It arises not only due to the larger amplitude of vibration, but also insensitivity to dissipation over a rather wide range. Therefore, provides a great economic impact for companies that make screens.

Figure 1 shows an example of a PR-screen test machine manufactured by a project partner in Poland [14]. Slepyan and Slepyan propose the dynamic model for a PR-vibrating screen [11]. The machine is modelled by the pre-tension of two equal masses, $M / 2$, connected by an elastic string with mass density per unit length, $\rho$, and string length $2 L$. The end masses are fixed in a rigid foundation by the side springs, $k$, which can move in the $u$ direction (Figure $1(\mathrm{~b})$ ).

The following two ordinary differential equations coupled by a geometric nonlinearity determine the screen motion:

$$
\begin{aligned}
& M \frac{d^{2} u(t)}{d t^{2}}+\beta \frac{d u(t)}{d t}+T_{1}(t)=Q \cos (\omega t+\varphi), \\
& \rho \frac{d^{2} w(t)}{d t^{2}}+\alpha \frac{d w(t)}{d t}+\frac{\pi^{2}}{4 l^{2}} T(t) w(t)=0,
\end{aligned}
$$

where $\beta$ and $\alpha$ are the viscosity numbers associated with the longitudinal and transverse oscillations, respectively, $u(t)$ is the displacement of the right end mass, and, therefore, $-u(t)$ corresponds to the left direction. $w(t)$ is the transverse displacement of point A (see Figure 1(b)) on the string in the first mode of vibration that depends only on time. The excitation signal is given by the excitation amplitude, $Q$, frequency, $\omega$, and $\varphi$ as the phase. The relation between tensile force, $T_{1}(t)$, and total force, $T(t)$, in the string is expressed by the following equations:

$$
\begin{aligned}
& T_{1}(t)=k\left(u(t)+\frac{\pi^{2}}{16 L} w^{2}(t)\right), \\
& T(t)=T_{0}+T_{1}(t)
\end{aligned}
$$

where $T_{0}$ is the initial tensile force. By replacing Eq. (2) in Eq. (1) and rearranging, the following equations arise:

$$
\begin{aligned}
M \frac{d^{2} u(t)}{d t^{2}} & +\beta \frac{d u(t)}{d t}+k u(t)=Q \cos (\omega t+\varphi) \\
& -\frac{k \pi^{2}}{16 L} w^{2}(t) \\
\rho \frac{d^{2} w(t)}{d t^{2}} & +\alpha \frac{d w(t)}{d t}+\frac{\pi^{2}}{4 L^{2}}\left(T_{0}+k u(t)\right) w(t) \\
& =\frac{k \pi^{2}}{64 L^{2}} w^{3}(t) .
\end{aligned}
$$

In fact, the parametric system in question consists of two nonlinear coupled subsystems where the primary system is externally excited by a harmonic force and the secondary system is parametrically excited with parametric excitation term, $\frac{\pi^{2}}{4 L^{2}}\left(T_{0}+k u(t)\right)$. Details of the screen dynamic model are presented in L. Slepyan and V. Slepyan [11].

To make the solution generic in the vibration analysis of the present study, simplified and more physically meaningful, the following dimensionless parameters are applied in this study:

$$
\begin{aligned}
& (\hat{u}, \hat{w})=\frac{(u, w)}{L}, \quad \hat{t}=t \sqrt{\frac{k}{M}}, \quad \hat{\omega}=\omega \sqrt{\frac{M}{k}}, \\
& \hat{\alpha}=\frac{\alpha}{\rho} \sqrt{\frac{M}{k}}, \quad \hat{\beta}=\frac{\beta}{\sqrt{k M}}, \\
& (\hat{T}(t), \hat{Q})=\frac{(T(t), Q)}{k L}, \quad \lambda=\frac{k L}{T_{0}} .
\end{aligned}
$$


Replacing the above dimensionless parameters in Eqs. (3), yields:

$$
\begin{aligned}
\frac{d^{2} \hat{u}(\hat{t})}{d \hat{t}^{2}} & +\hat{\beta} \frac{d \hat{u}(\hat{t})}{d \hat{t}}+\hat{u}(\hat{t})=\hat{Q} \cos (\hat{\omega} \hat{t}+\varphi) \\
& -\frac{\pi^{2}}{16} \hat{w}^{2}(\hat{t}) \\
\frac{d^{2} \hat{w}(\hat{t})}{d \hat{t}^{2}} & +\hat{\alpha} \frac{d \hat{w}(\hat{t})}{d \hat{t}}+\hat{\Omega}_{T}^{2}[1 \\
& \left.+\lambda\left(\hat{u}(\hat{t})+\frac{\pi^{2}}{16} \hat{w}^{2}(\hat{t})\right)\right] \hat{w}(\hat{t})=0
\end{aligned}
$$

where $\hat{\Omega}_{T}=\frac{\pi}{2 L} c_{T}$ is the frequency of the longitudinal small-amplitude free oscillations and $c_{T}=\sqrt{\frac{T_{0}}{\rho}}$ is the transverse wave speed in the string. To run the new design of the vibrating screen, the domain of the optimal parameters needs to be obtained. This subject is an interesting mathematical model, as the functional parametric equations are inherently nonlinear and do not have a closed-form analytical solution. The calculated domain should avoid divergence or inappropriate results in the machine's running life. One well-known analytical practice to solve nonlinear equations is the perturbation method. Several techniques in this family, such as the Variation Iteration Method (VIM) [1519], the Homotopy Perturbation Method (HPM) [2024], the Homotopy Analysis Method (HAM) [25-27], and multi-step differential transform method [28] have been developed to solve nonlinear equations. In this research, HPM is applied to derive an explicit solution of the screen equations. The analytical model developed can be used in other PR applications, although this is probably the largest machine available at this time.

The paper is organized as follows. In Section 2, the fundamental formulations of HPM are introduced to solve nonlinear parametric equations, followed by analytical solutions and the stability domains of the screen under impact and non-impact operation conditions in Section 3. Section 4 summarises the research.

\section{Concept of HPM}

The formulation of HPM is illustrated by the following nonlinear differential equation:

$$
A(u)=f(r), r \in \Omega,
$$

with boundary conditions:

$$
B\left(u, \frac{\partial u}{\partial n}\right)=0, \quad r \in \Gamma,
$$

where $A$ is a general differential operator, $B$ notes a boundary operator, $f(r)$ is a known analytical function, and $\Gamma$ represents the boundary of the domain, $\Omega$. The operator, $A$, is divided into two parts, $L$ (linear) and $N$ (nonlinear). Therefore, Eq. (6) is rewritten as:

$$
L(u)+N(u)=f(r) .
$$

A homotopy perturbation form is built, $v(r, p): \Omega \times$ $[0,1] \rightarrow R$, which satisfies [18]:

$$
\begin{aligned}
& H(v, p)=(1-p)\left[L(v)-L\left(u_{0}\right)\right]+p[A(v)-f(r)]=0, \\
& \quad p \in[0,1], \quad r \in \Omega,
\end{aligned}
$$

where $p \in[0,1]$ is an embedding parameter and $u_{0}$ is an initial approximation of Eq. (6) which satisfies the boundary condition. Therefore:

$$
\left\{\begin{array}{l}
H(v, 0)=L(v)-l\left(u_{0}\right)=0 \\
H(v, 1)=A(v)-f(r)=0
\end{array}\right.
$$

The changing of the $p$ parameter from zero to unity leads to $v(r, p)$ moving from $u_{0}(r)$ to $u(r)$. According to HPM, role $p$ is embedded as a small parameter, and the solution of Eq. (8) is expressed as a power series in $p$, as follows:

$$
v=v_{0}+p v_{1}+p^{2} v_{2}+\cdots .
$$

The approximate solution when $p \rightarrow 1$ in Eq. (10) is:

$$
u=\lim _{p \rightarrow 1} v=v_{0}+p v_{1}+p^{2} v_{2}+\cdots
$$

In fact, the combination of the perturbation method and homotopy technique is called the HPM and remedies the limitations of the traditional perturbation methods [20]. The convergence rate of HPM, which comes from convergence of series (Eq. (8)), depends on the nonlinear operator $A(v)$ [25]. To date, some interesting results have been published based on this method [20-25,28-31]. Turkyilmazoglu [30] has shown that under particular choices of auxiliary parameters, the HPM is indeed the Taylor series expansion of the sought solution of nonlinear equations. In this research, Maple software is used to find closed-form solutions of $u(t)$ and $w(t)$ as a function of the variable parameters to estimate the parametric resonant domains.

\section{Parametric Resonance (PR) domains}

PR has the ability to exhibit higher mechanical amplification than direct resonance. While, in most resonance-related research, the motivation is to develop techniques to avoid or minimize the damaging effects of PR on the structure operating within the expected 
range of excitation frequencies and forces, in this study, the PR oscillations try to create a well-ordered and stable regime. Zahedi and Babitsky [12] determined the appropriate harmonic PR for the particular system chosen occurred at a frequency of $1 \mathrm{~s}^{-1}$. The natural frequency of the sieve plate is around $0.5 \mathrm{~s}^{-1}$, while the external excitation frequency is around $1 \mathrm{~s}^{-1}$. Therefore, while the first system vibrates in normal resonance mode, the second operates at twice its natural frequency (see Eq. (5)).

Calculating the boundaries of the PR domain analytically in the PR-based screen machine is a challenging subject due to the nonlinear terms in Eq. (5). In this section, HPM is applied as a powerful computational technique to determine the domain of unstable response with variable working parameters. Both non-impact and impact working conditions are studied thoroughly in this section.

It is worth mentioning that the HPM solution, however, derives for symbolic initial conditions, and the paper is written based on specific values for initial parameters. The reason is that $\hat{u}(\hat{t})$ and $\hat{w}(\hat{t})$ expressions in some cases extend to fill an entire sheet. Both initial and boundary conditions used in this manuscript, picked up from [10,12], demonstrate a new PR-based screen machine.

\subsection{Non-impact analysis}

The maximum weight of material placed on the sieve plate and the mesh weight itself determine the $\rho$ parameter in Eq. (5). The first case study is to assume $\rho$ is constant and the screen machine is modelled under non-impact working conditions. The homotopy equation is written in the following form:

$$
\begin{aligned}
H P_{1}= & (1-p)\left(\frac{d^{2} \hat{u}(\hat{t})}{d \hat{t}^{2}}+\hat{u}(\hat{t})\right) \\
& +p\left(\frac{d^{2} \hat{u}(\hat{t})}{d \hat{t}^{2}}+\hat{\beta} \frac{d \hat{u}(\hat{t})}{d \hat{t}}+\hat{u}(\hat{t})\right. \\
& \left.-\hat{Q} \cos (\hat{\omega} \hat{t}+\varphi)+\frac{\pi^{2}}{16} \hat{w}^{2}(\hat{t})\right)=0 \\
H P_{2}= & (1-p)\left(\frac{d^{2} \hat{w}(\hat{t})}{d \hat{t}^{2}}+\hat{\Omega}_{T}^{2} \hat{w}(\hat{t})\right) \\
& +p\left(\frac{d^{2} \hat{w}(\hat{t})}{d \hat{t}^{2}}+\hat{\alpha} \frac{d \hat{w}(\hat{t})}{d \hat{t}}+\hat{\Omega}_{T}^{2}[1\right. \\
& \left.\left.+\lambda\left(\hat{u}(\hat{t})+\frac{\pi^{2}}{16} \hat{w}^{2}(\hat{t})\right)\right] \hat{w}(\hat{t})\right)=0
\end{aligned}
$$

with the following initial conditions:

$$
\begin{aligned}
& \left.\hat{u}(0)=0.0025, \frac{d \hat{u}(\hat{t})}{d \hat{t}}\right)_{\hat{t}=0}=0, \\
& \left.\hat{w}(0)=-0.06, \frac{d \hat{w}(\hat{t})}{d \hat{t}}\right)_{\hat{t}=0}=0 .
\end{aligned}
$$

By setting $p=0$ in $H P_{1}$ and $H P_{2}$ in Eqs. (12) and (13), they become linear and $p=1$ brings them into the original nonlinear form. It continuously traces an implicitly defined curve from starting point $H(\hat{u}, 0)$ and $H(\hat{w}, 0)$ to solution functions $H(\hat{u}, 1)$ and $H(\hat{w}, 1)$. According to HPM, the closed-form solution of $\hat{u}(r)$ and $\hat{w}(r)$ is expressed in a series of $p$ :

$$
\begin{aligned}
& \hat{u}(r)=\hat{u}_{0}(r)+p \hat{u}_{1}(r)+p^{2} \hat{u}_{2}(r)+\cdots \\
& \hat{w}(r)=\hat{w}_{0}(r)+p \hat{w}_{1}(r)+p^{2} \hat{w}_{2}(r)+\cdots
\end{aligned}
$$

Substituting $\hat{u}(r)$ from Eq. (15) into Eq. (12), and equating the terms with identical powers of $p$ :

$$
\begin{aligned}
p^{0}: \quad \frac{d^{2} \hat{u}_{0}(\hat{t})}{d \hat{t}^{2}} & +\hat{u}_{0}(\hat{t})=0 \\
p^{1}: \quad \frac{d^{2} \hat{u}_{1}(\hat{t})}{d \hat{t}^{2}} & +\hat{u}_{1}(\hat{t})-\frac{1}{1200} \times \cos (\hat{t})+\frac{1}{150} \\
& \times \frac{d \hat{u}_{0}(\hat{t})}{d \hat{t}}+\frac{1}{16} \pi^{2} \hat{w}_{0}(\hat{t})^{2}=0, \\
p^{2}: \quad \frac{d^{2} \hat{u}_{2}(\hat{t})}{d \hat{t}^{2}} & +\hat{u}_{2}(\hat{t})+\frac{1}{150} \frac{d \hat{u}_{1}(\hat{t})}{d \hat{t}}+\frac{1}{18} \pi^{2} \\
& \times \hat{w}_{0}(\hat{t}) \times \hat{w}_{1}(\hat{t})=0 .
\end{aligned}
$$

Substituting $\hat{w}(r)$ from Eq. (16) into Eq. (13), and equating the terms with identical powers of $p$, repeated for $\hat{w}(r)$, gives the following equations:

$$
\begin{aligned}
& p^{0}: \frac{d^{2} \hat{w}_{0}(\hat{t})}{d \hat{t}^{2}}+0.2467 \times \hat{w}_{0}(\hat{t})=0 \\
& p^{1}: \quad \frac{d^{2} \hat{w}_{1}(\hat{t})}{d \hat{t}^{2}}+0.2464 \times \hat{w}_{1}(\hat{t})+6.168 \\
& \quad \times \pi^{2} \times \hat{w}_{0}(\hat{t})^{3}-0.1333 \times \frac{d \hat{w}_{0}(\hat{t})}{d \hat{t}} \\
& \quad+98.696 \times \hat{w}_{0}(\hat{t}) \times \hat{u}_{0}(\hat{t})=0 \\
& p^{2}: \quad \frac{d^{2} \hat{w}_{2}(\hat{t})}{d \hat{t}^{2}}+0.250 \times \hat{w}_{2}(\hat{t})-0.250 \\
& \quad \times \hat{w}_{1}(\hat{t})-0.130 \times \frac{d \hat{w}_{1}(\hat{t})}{d \hat{t}}+0.25 \\
& \quad \times\left(1+394.8 \times \hat{u}_{0}(\hat{t})+24.675 \times \pi^{2} \times \hat{w}_{0}(\hat{t})^{2}\right)
\end{aligned}
$$




$$
\begin{aligned}
& \times \hat{w}_{1}(\hat{t})+0.250 \times\left(\left(394.8 \times \hat{u}_{1}(\hat{t})+49.35\right.\right. \\
& \left.\left.\times \pi^{2} \times \hat{w}_{0}(\hat{t}) \times \hat{w}_{1}(\hat{t})\right) \times \hat{w}_{0}(\hat{t})\right)=0 .
\end{aligned}
$$

According to the predefined initial conditions (Eq. (14)), the initial approximations for $\hat{u}_{0}(\hat{t})$ and $\hat{w}_{0}(\hat{t})$ are given as:

$$
\begin{aligned}
& \hat{u}_{0}(\hat{t})=\frac{1}{400} \cos (\hat{t}), \\
& \hat{w}_{0}(\hat{t})=-\frac{3}{50} \cos \left(\frac{1}{10^{4}} \sqrt{2.46 \times 10^{7}} \hat{t}\right) .
\end{aligned}
$$

By solving Eqs. (18) and (21) based on the initial approximations, Eqs. $(23)$ and $(24), \hat{u}_{1}(\hat{t})$ and $\hat{w}_{1}(\hat{t})$ are revealed to be:

$$
\begin{aligned}
& \hat{u}_{1}(\hat{t})=8.3 \times 10^{6} \times \sin (\hat{t})+0.086 \times \cos (\hat{t}) \\
& +4.16 \times 10^{4} \times \sin (\hat{t}) \times \hat{t}-0.085 \times \cos (0.993 \hat{t}) \\
& \quad-0.001-8.33 \times 10^{7} \times \cos (\hat{t}) \times \hat{t} \\
& \hat{w}_{1}(\hat{t})=1.1 \times \cos (0.496 \hat{t})-1.131 \times \cos (0.503 \hat{t}) \\
& \quad-0.003 \times \cos (1.496 \hat{t})-0.001 \times \cos (1.490 \hat{t})
\end{aligned}
$$

After carrying out the procedure, the rest of the components, $\hat{u}_{2}, \hat{u}_{3}, \cdots$ and $\hat{w}_{2}, \hat{w}_{3}, \cdots$, are obtained.

Finally, according to HPM, the closed-form solutions of longitudinal and transverse displacement are:

$$
\begin{aligned}
\hat{u}(\hat{t}) & =\sum \hat{u}_{i}(\hat{t}), \quad i=0,1,2, \cdots, \\
\hat{w}(\hat{t}) & =\sum \hat{w}_{i}(\hat{t}), \quad i=0,1,2, \cdots .
\end{aligned}
$$

Figure 2 shows the amplitude-time response for one cycle of oscillation of $\hat{u}(\hat{t})$ and $\hat{w}(\hat{t})$, solved by HPM and the Runge Kutta 4th order (RK4) numerical method. The HPM approximate series of the longitudinal $\hat{u}(\hat{t})$

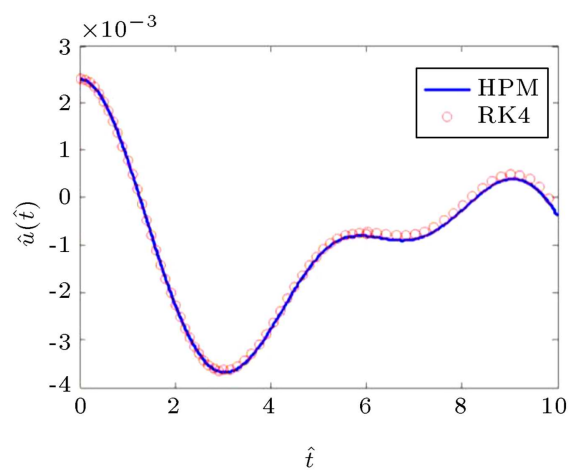

(a) and transverse $\hat{w}(\hat{t})$ displacements are truncated with three terms, as the HPM results are in good agreement (in tolerance of $0.1 \%$ ) with the RK4 numerical method. Further accuracy can be reached by using higher computational time. The blue line depicts HPM and RK4 is represented by the hollow circles. The values $\Omega_{L}=1, \Omega_{T}=\frac{1}{2}, \hat{\alpha}=\frac{2}{15}, \hat{\beta}=\frac{1}{150}, \mu=0.025$ and $\lambda=400$ are set in Figure 2 .

A particular feature of the parametric screen machine is that for certain values of excitation frequency $(\hat{Q})$ and certain values of working parameters, the primary system has an oscillating response, whereas the secondary system is at rest. When the oscillations of the primary system produce oscillating behaviour in the secondary subsystem, the solution becomes stable. Therefore, for a given pair of parameter values, the system can exhibit bounded (stable) or unbounded (unstable) solutions. In this study, the amplitude PRstable regime is considered in the ranges $0.01<\hat{u}(\hat{t})<$ 0.04 and $0.02<\hat{w}(\hat{t})<0.1[12]$.

These ranges are set to avoid damage to the screen mesh and increase its longevity. Outside these domains, the oscillations of the secondary system grow unbounded, potentially leading to catastrophic failure of the screen or mechanical breakdown.

A key point in setting up the machine is to carefully delineate the parameter space in which the stable PR modes are found. Since no explicit solution is given for the stability of this screen machine, the homotopy solution is applied. It is common in stability analysis to show a stable operation diagram where the stability regions are presented. The transition curves shown in Figure 3 separate the stable (white) from the unstable (grey) regions of PR. Note that the horizontal axis is frequency ratio $\left(r=\omega / \omega_{n}\right)$. The results obtained allow for refinement of the domains of optimal parameters, where the parametric oscillations are excited and where the analytically-obtained steady oscillation regimes are stable. Figure 3 shows that the main stability domain corresponds to the 2:1 parametric excitation. The

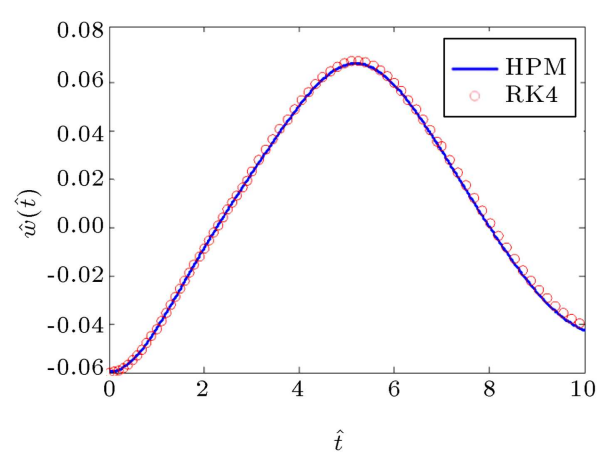

(b)

Figure 2. Longitudinal oscillation $\hat{u}(\hat{t})$ (a) and transverse oscillations $\hat{w}(\hat{t})$ (b) with $\Omega_{L}=1, \Omega_{T}=\frac{1}{2}, \hat{\alpha}=\frac{2}{15}, \hat{\beta}=\frac{1}{150}$, $\mu=0.025$ and $\lambda=400$. 


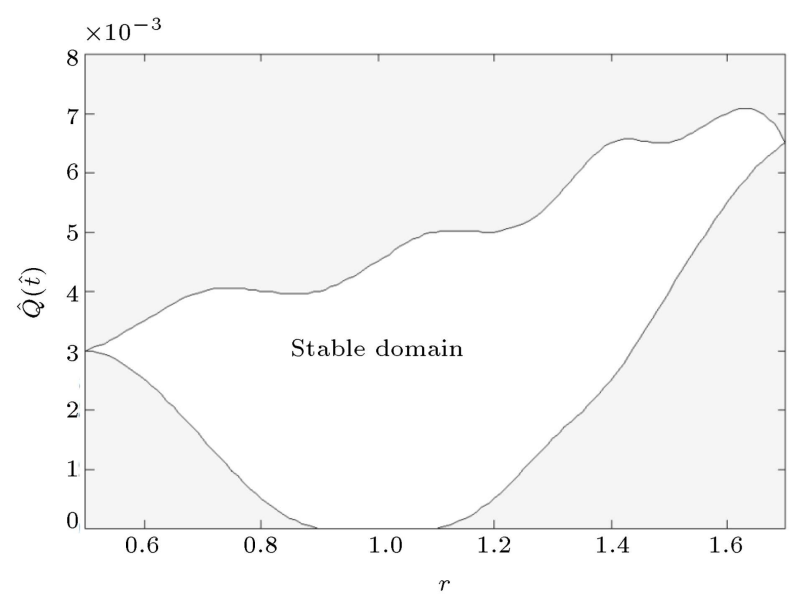

Figure 3. Stable boundaries of screen machine $\left(\Omega_{L}=1\right.$, $\Omega_{T}=\frac{1}{2}, \hat{\alpha}=\frac{2}{15}, \hat{\beta}=\frac{1}{150}, \mu=0.025$ and $\left.\lambda=400\right)$.

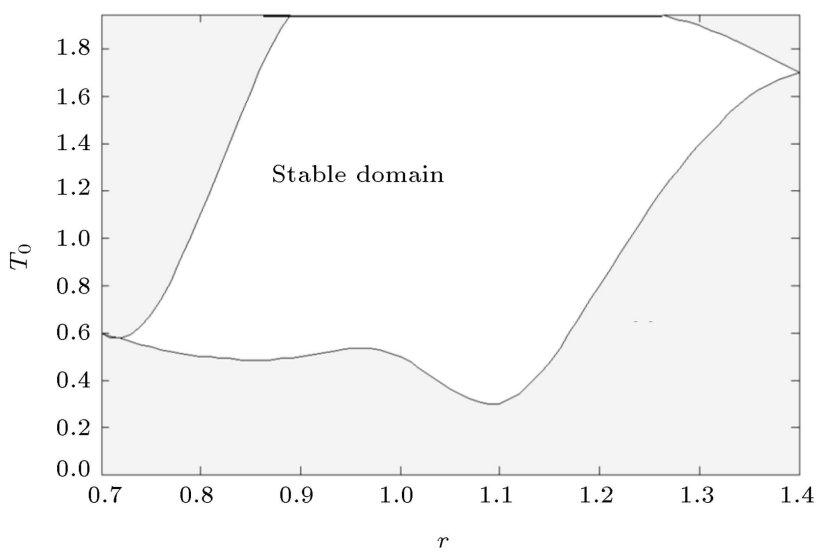

Figure 4. Stability diagram under different forces $\left(\Omega_{L}=1, \Omega_{T}=\frac{1}{2}, \hat{\alpha}=\frac{2}{15}, \hat{\beta}=\frac{1}{150}, \mu=0.025\right.$ and $\lambda=400)$.

following parameters are used to produce this figure: $\Omega_{L}=1, \Omega_{T}=\frac{1}{2}, \hat{\alpha}=\frac{2}{15}, \hat{\beta}=\frac{1}{150}, \mu=0.025$ and $\lambda=400$.

The screen machine needs to stretch initially by $T_{0}$. Figure 4 shows the stability diagram of the screen machine under different initial tensile forces. This figure gives a good indication of the direction the $T_{0}$ parameter needs to acquire to get into the stable region. It is essential for operators to require as little manual intervention as possible to bring the system into the stable domain.

\subsection{Impact analysis}

The flow of treated materials does not generally have a constant weight as the screen system oscillates. In such situations, the general mechanical modelling has to be properly modified. The finite discontinue mass variation over a very short time strongly affects the tuning of the PR domain. In this research, a constant rate of mass change is assumed. The motion is described with differential equations with variable mass parameters. The equivalent mass per unit length $\rho$ varies between $10 \mathrm{~kg} / \mathrm{m}$ and $12 \mathrm{~kg} / \mathrm{m}$ in the regimes of oscillation with periodic interruption of contact (vibroimpact regime). Eqs. (28) trace the influence of the screen structure, setting, granular material weight and linear effective viscosity. In this case, the screen moves with a downward or upward trajectory with high values of acceleration, $20 \%$ of the mass of the material is considered to separate and leave the screen in one cycle. By changing the $\rho$ parameter between 10 and $12 \mathrm{~kg} / \mathrm{m}$ for each cycling motion, more realistic boundary conditions between the screen and the treated material are taken into account:

$$
\begin{aligned}
\frac{d^{2} \hat{u}(\hat{t})}{d \hat{t}^{2}} & +\hat{\beta} \frac{d \hat{u}(\hat{t})}{d \hat{t}}+\hat{u}(\hat{t})=\hat{Q} \cos (\hat{\omega} \hat{t}+\varphi) \\
& -\frac{\pi^{2}}{16} \hat{w}^{2}(\hat{t}) \\
\frac{d^{2} \hat{w}(\hat{t})}{d \hat{t}^{2}} & +\hat{\alpha} \frac{d \hat{w}(\hat{t})}{d \hat{t}}+\hat{\Omega}_{T}^{2}\left[1+\lambda\left(\hat{u}(\hat{t})+\frac{\pi^{2}}{16} \hat{w}^{2}(\hat{t})\right)\right] \hat{w}(\hat{t}) \\
& =0 .
\end{aligned}
$$

The HPM method is applied to solve Eqs. (28) when the mass is varied continuously over time. Figure 5 shows the boundaries of the PR stable domain based on the vibro-impact excitation condition. Since dynamic vibro-impact response is established over a wide frequency range, the analytical solution of HPM provides appropriate information to set up the screen machine accurately. Figure 5 shows that the stability domain of the machine under vibro-impact operation is more limited than under the non-impact boundary condition. Figure 6 shows the effect of the initial tensile force under the vibro-impact mechanism. The following operation process parameters are used to plot Figure 6 . Note that this figure is only valid for $\Omega_{L}=1, \Omega_{T}=\frac{1}{2}$, $\hat{\alpha}=\frac{2}{15}, \hat{\beta}=\frac{1}{150}, \mu=0.025$ and $\lambda=400$. The

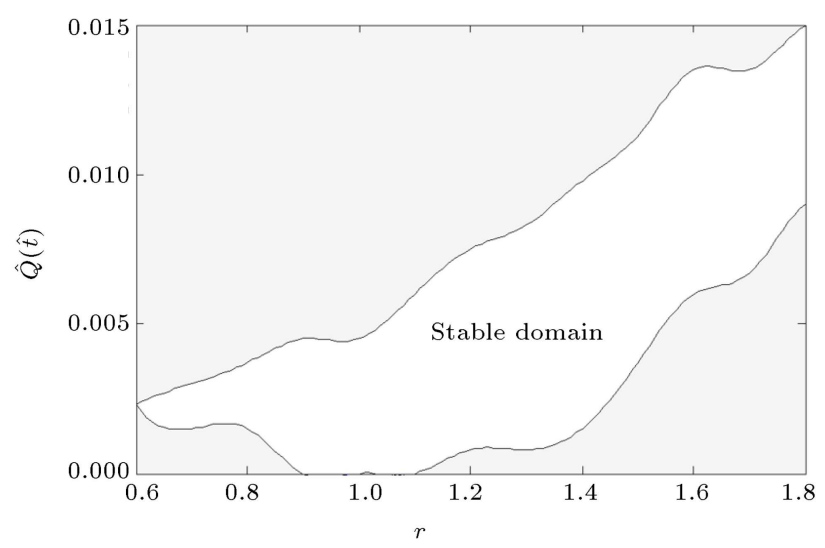

Figure 5. Stability diagram of screen machine based on vibro-impact excitation type. 


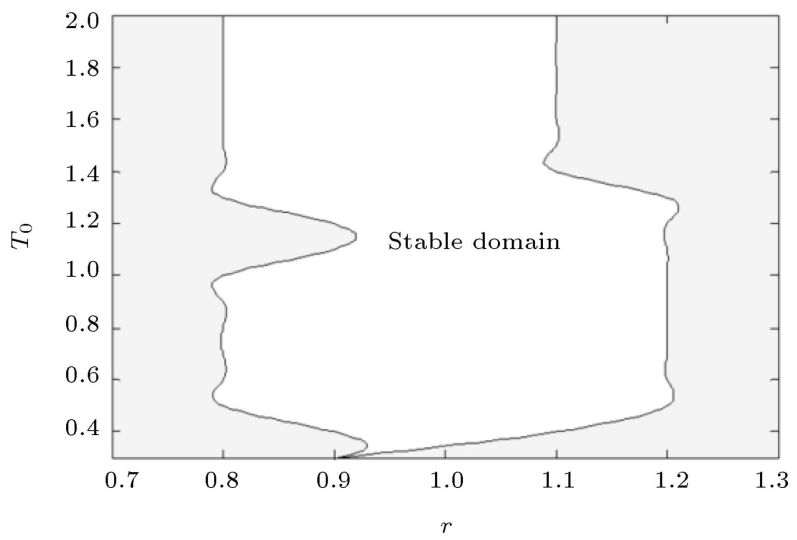

Figure 6. Stability diagram under different forces.

approximate analytical solution of HPM can easily be extended for different values of the parameters.

In this study, the stability regions are investigated and shown in shaded figures (Figures 3-6). These figures provide a good indication of the screen mesh behaviour for different operation parameters and boundary conditions. Since the stability regions are well defined, it is easy to see how much a certain parameter needs to be increased to move outside the stable region. The consequences of a vibrating screen and reconfigurable screen failure have a direct negative economic effect through production losses, repair costs and eventual losses resulting from stopping the work of other machines in the conditioned technological chain. Therefore, companies look to properly use the screen mesh and maintain it in order to maximise their productivity and efficiency. The results of this paper have a direct impact on reducing some of the uncertainty in the decisions made concerning screen mesh.

\section{Concluding remarks}

Sieving is one of the oldest physical size separation methods and is used widely in both industry and in laboratories. The PR-based screen machine is a new invention that has a great practical application to increase sieving efficiency. To control screen mesh motion, it is important to know the influences of certain operational parameters on the stability of the structure. In this paper, Homotopy Pertubation Method (HPM) is successfully applied to find the stable boundaries of PR-based screening machines that separate or classify mixtures of solid particles of different sizes. Since the weight of the materials treated is usually comparable with the mass of the screen mesh, the HPM method is used to solve both non-impact and impact excitation motions. The results show that HPM provides excellent approximations of the solution of this nonlinear system with high accuracy. HPM presents a rapid convergence of solutions without the difficulties that arise from traditional analytical methods. This method has the capability for a wide-range of applications in nonlinear systems. Further research is required in order to obtain precise knowledge of the damping mechanisms during impact.

\section{Nomenclature}

A General differential operator

$B \quad$ Notes a boundary operator

$c_{T} \quad$ Transverse wave speed in the string

$f(r) \quad$ Known analytical function

HAM Homotopy Analysis Method

HPM Homotopy Perturbation Method

$k \quad$ Rigid foundation by the side springs

$L \quad$ String length

$L(u) \quad$ Linear part of differential equation

$M \quad$ Mass

$N(u) \quad$ Nonlinear part of differential equation

$p \quad$ Homotopy parameter

PR Parametric Resonance

$Q \quad$ Excitation amplitude

RK4 Runge Kutta 4th order

$t \quad$ Time

$T(t) \quad$ Total force

$T_{0} \quad$ Initial tensile force

$T_{1}(t) \quad$ Tensile force

$u(t) \quad$ Displacement of mass

VIM Variation Iteration Method

$w(t) \quad$ Transverse displacement

$\alpha \quad$ Transverse viscosity number

$\beta \quad$ Longitudinal viscosity number

$\Gamma \quad$ Boundary of the domain $\Omega$

$\rho \quad$ Density per unit length

$\omega \quad$ Frequency

$\hat{\Omega}_{T} \quad$ Frequency of the longitudinal small-amplitude free oscillations

$\varphi \quad$ Phase

\section{References}

1. Zahedi, S.A., Roy, A., and Silberschmidt, V.V. "Modelling of vibration assisted machining fcc single crystal", Procedia CIRP, 31, pp. 393-398 (2015).

2. Zahedi, S.A., Roy, A., and Silberschmidt, V.V. "Vibration-assisted machining of single crystal", Journal of Physics: Conference Series, 451(1), pp. 1203812043 (2013). 
3. Makinde, O.A., Ramatsetse, B.I., and Mpofu, K. "Review of vibrating screen development trends: Linking the past and the future in mining machinery industries", International Journal of Mineral Processing, 145, pp. 17-22 (2015).

4. Xiao-mei, H.E. and Chu-sheng, L. "Dynamics and screening characteristics of a vibrating screen with variable elliptical trace", Mining Science and Technology, 19, pp. 508-513 (2009).

5. Djoković, J.M., Tanikić, D.I., Nikolić, R.R., et al. "Screening efficiency analysis of vibrosieves with the circular vibrations", Civil and Environmental Engineering, 13(1), pp. 77-83 (2017).

6. Liu, K.S. "Some factors affecting sieving performance and efficiency", Powder Technology, 193(2), pp. 208213 (2009).

7. Baragetti, S. and Villa, F. "A dynamic optimization theoretical method for heavy loaded vibrating screens", Nonlinear Dynamics, 78(1), pp. 609-627 (2014).

8. Liu, A., Lu, H., Fu, J., et al. "Analytical and experimental studies on out-of-plane dynamic instability of shallow circular arch based on parametric resonance", Nonlinear Dynamics, 87(1), pp. 677-694 (2017).

9. Amorim, T.D., Dantas, W.G., and Gusso, A. "Analysis of the chaotic regime of MEMS/NEMS fixed-fixed beam resonators using an improved 1DOF model", Nonlinear Dynamics, 79(2), pp. 967-981 (2015).

10. Shmavonyan, G.Sh. and Zendehbad, S.M. "Treatment of $\mathrm{ZnO}$ nanowires on $\mathrm{Si}$ (111)", Scientia Iranica, 18(3), pp. 816-819 (2011).

11. Slepyan, L. and Slepyan, V. "Coupled model parametric resonance in a vibrating screen", Mechanical Systems and Signal Processing, 43, pp. 295-304 (2014).

12. Zahedi, S.A. and Babitsky, V. "Modeling of autoresonant control of a parametrically excited screen machine", Journal of Sound and Vibration, 380, pp. 7889 (2016).

13. Zahedi, S.A. and Babitsky, V. "Autoresonant excitation and control of parametric vibration", NOC Conference, Budapest (2017).

14. Bkak, Ł., Noga, S., and Stachowicz, F. "Modelling and numerical simulation of parametric resonance phenomenon in vibrating screen", Vibrations in Physical Systems, 27 (2016).

15. Kazemnia, M., Zahedi, S.A., Vaezi, M., et al. "Assessment of modified variational iteration method in BVPs high-order differential equations", Journal of Applied Sciences, 8(22), pp. 4192-4197 (2008).

16. Zahedi, S.A., Demiral, M., Roy, A., et al. "FE/SPH modelling of orthogonal micro-machining of f.c.c. single crystal", Computational Materials Science, 78, pp. 104-109 (2013).

17. Turkyilmazoglu, M. "An optimal variational iteration method", Applied Mathematics Letters, 24, pp. 762765 (2011).
18. Kazeminia, M., Soleimani-Amiri, S., and Zahedi, S.A. "Exact and numerical solutions for nonlinear higher order modified $\mathrm{KdV}$ equations by using variational iteration method", Advanced Studies in Theoretical Physics, 4(9), pp. 437-447 (2010).

19. Zahedi, S.A., Goodarzian, H., and Okazi, M. "An investigation on two-dimensional non-linear wave equation using VIM", Indian Journal of Science and Technology, 3(9) (2010).

20. Fazeli, M., Zahedi, S.A., and Tolou, N. "Explicit solution of nonlinear fourth-order parabolic equations via homotopy perturbation method", Journal of Applied Sciences, 8, pp. 2619-2624 (2008).

21. Gupta, P.K., Singh, M., and Yildirim, A. "Approximate analytical solution of the timefractional Camassa-Holm, modified Camassa-Holm, and Degasperis-Procesi equations by homotopy perturbation method", Scientia Iranica, 23(1), pp. 155-165 (2016).

22. Fereidoon, A., Zahedi, S.A., Ganji, D.D., and Rostamiyan, Y. "Homotopy Perturbation method to solving nonlinear WBK equations", Far East Journal of Dynamical Systems, 10(2), pp. 239-254 (2008).

23. Khatami, I. and Zahedi, M. "Nonlinear vibration analysis of axially moving string", SN Applied Sciences, 1(12), pp. 1668-1698 (2019).

24. Turkyilmazoglu, M. "An effective approach for evaluation of the optimal convergence control parameter in the homotopy analysis method", Filomat, 30(6), pp. 1633-1650 (2016).

25. Turkyilmazoglu, M. "Approximate analytical solution of the nonlinear system of differential equations having asymptotically stable equilibrium", Filomat, 31(9), pp. 2633-2641 (2017).

26. Zahedi, S.A., Fazeli, M., and Tolou, N. "Analytical solution of time-dependent non-linear partial differential equations using HAM, HPM and VIM", Journal of Applied Sciences, 8(16), pp. 2888-2894 (2008).

27. Khatami, I., Zahedi, M., and Zahedi, E. "Efficient solution of nonlinear Duffing oscillator", Journal of Applied and Computational Mechanics, 6(2), pp. 219234 (2020).

28. Khatami, I., Pashai, M.H., and Tolou, N. "Comparative vibration analysis of a parametrically nonlinear excited oscillator using HPM and numerical method", Mathematical Problems in Engineering, 2008, Article ID 956170 (2008).

29. Roy, P.K., Das, A., Mondal, H., et al. "Application of homotopy perturbation method for a conductiveconvective fin with temperature dependent thermal conductivity and surface emissivity", Ain Shams Engineering Journal, 6, pp. 1001-1008 (2015).

30. Turkyilmazoglu, M. "Is homotopy perturbation method the traditional Taylor series expansion", Hacettepe Journal of Mathematics and Statistics, 44(3), pp. 651-665 (2015). 
31. Roy, P.K. and Mallick, A. "Thermal analysis of straight rectangular fin using homotopy perturbation method", Alexzandria Engineering Journals, 55, pp. $2269-2277$ (2016).

\section{Biographies}

Mohsen Zahedi is an MS student in Computer Engineering at Shiraz University in Iran. He awarded his BS degree (2017) in Computer Engineering from Isfahan University, Iran, with distinction. He has a substantial background in artificial intelligence, data analysis, computational modelling, and has versatile knowledge and experience in signal processing.

Iman Khatami is Assistant Professor in Mechanical Engineering at Chabahar Maritime University in Iran. He was a doctorate candidate and research assistant at Universite de Sherbrooke in Canada (2009-2014). His $\mathrm{PhD}$ research program was conducted in free filed noise identification of aircraft engines using circular array. In 2014-2015, Dr. Khatami was awarded a postdoctoral position at Universite de Sherbrooke and worked closely in the Laboratory with the Intelligent Vehicles
(LIV) research team. He has authored and co-authored several journal papers in his fields of interest, which include acoustics and vibration, signal processing, and intelligent systems applied to automotives.

Seyed Abolfazl Zahedi was awarded his PhD (2013) in Mechanical Engineering from Loughborough University, UK. Following the completion of his $\mathrm{PhD}$, he received a Marie Curie research fellowship, which allowed him to extend his collaboration with the research group at Loughborough University in the mechanics of advanced materials. He subsequently joined the Advance Forming Research Centre (AFRC) at the University of Strathclyde as a research associate. Professor Zahedi was appointed research fellow in the school of Mechanical, Aerospace and Civil Engineering (MACE) at the University of Manchester in 2015 and has since become senior lecturer at De Montfort University, UK. His main research interests include computational material modelling, additive manufacturing, tissue engineering, and vibration analysis. He is interested in using applied mathematics to solve engineering problems, particularly analytical methods to facilitate design. 